\title{
Padovan Numbers by the Permanents of a Certain Complex Pentadiagonal Matrix
}

\author{
Diyar O.Mustafa Zangana ${ }^{1}$ Ahmet Öteleş ${ }^{2}$ \\ ${ }^{1}$ Siirt University, Faculty of Science, Department of Mathematics, TR-56100, Siirt, Turkey \\ diyar.math2@gmail.com \\ ${ }^{2}$ Dicle University, Education Faculty, Department of Mathematics, TR-21280, Diyarbakir/Turkey \\ aoteles85@gmail.com
}

\section{Abstract}

In this paper, we consider a certain type of complex pentadiagonal matrices. Then we show that the permanents of this matrix generate Padovan numbers. Finally, we give a Maple procedure in order to verify our result.

Keywords: Permanent, Pentadiagonal matrix, Padovan number.

\section{Introduction}

The famous integer sequences (e.g., Fibonacci, Lucas, Padovan) provide invaluable opportunities for exploration, and contribute handsomely to the beauty of mathematics, especially number theory [1, 2]. Among these sequences, Padovan numbers have achieved a kind of celebrity status. The Padovan sequence $\{P(n)\}$ is defined by the recurrence relation, for $\mathrm{n}>2$

$$
P(n)=P(n-2)+P(n-3)
$$

with $\mathrm{P}(0)=\mathrm{P}(1)=\mathrm{P}(2)=1[3]$. The number $\mathrm{P}(\mathrm{n})$ is called $\mathrm{n}^{\text {th }}$ Padovan number. The Padovan numbers are

$$
1,1,1,2,2,3,4,5,7,9,12,16,21,28,37,49, \ldots
$$

for $\mathrm{n}=0,1,2, \ldots$ This sequence is named as A000931 in [4].

The permanent of a $\mathrm{n} \times \mathrm{n}$ matrixA $=\left(\mathrm{a}_{\mathrm{ij}}\right)$ is defined by 


$$
\operatorname{Per}(\mathrm{A})=\sum_{\sigma \in \mathrm{S}_{\mathrm{n}}} \prod_{\mathrm{i}=1}^{\mathrm{n}} \mathrm{a}_{\mathrm{i} \sigma}(\mathrm{i})
$$

where the summation extends over all permutations $\sigma$ of the symmetric group $S_{n}$. Thepermanent of a matrix is analogous to the determinant, where all of the signs used in theLaplace expansion of minors are positive.

Permanents have many applications in physics, chemistry, graph theory, electrical engineering, and so on [5, 6, 7, 8,9]. One of the most important applications of

permanents is the relationship between some special types of matrices and the wellknown number sequences. There are many papers in relation to that applications. $[10,11$, $12,13,14,15,16,17,18,19,20,21,22,23,24]$ are some of them.

In this paper, we consider a certain type of complex pentadiagonal matrices. Then we show that the permanents of this matrix generate Padovan numbers. Finally, we give a Maple procedure in order to verify our result.

\section{Main Results}

Let $A=\left[a_{i j}\right]$ be an $m \times n$ real matrix with row vectors $a_{1}, a_{2}, \ldots, a_{m}$. We say $A$ iscontractible on column(resp. row) $\mathrm{k}$ if column (resp. row) kcontains exactly twononzero entries. Suppose $A$ is contractibleon column k with $a_{i k} \neq 0 \neq a_{j k}$ and $i \neq j$. Then the $(m-1) \times(n-1)$ matrix $A_{i j: k}$ obtained fromA by replacing row $i$ with $\mathrm{a}_{\mathrm{jk}} \alpha_{\mathrm{i}}+\mathrm{a}_{\mathrm{ik}} \alpha_{\mathrm{j}}$ and deleting row $\mathrm{j}$ and column $\mathrm{k}$ is called the contraction of $\mathrm{A}$ on column $\mathrm{k}$ relative to rows $i$ and $j$. If $A$ is contractible on row $a_{k i} \neq 0 \neq a_{k j}$ and $i \neq j$, then the matrix $A_{k: i j}=\left[A_{i j: k}^{T}\right]^{T}$ is called the contraction of $A$ on row k relative to columns $i$ and $j$. We say that $A$ can be contracted to a matrix $B$ if either $B=A$ or there exist matrices $A_{0}, A_{1}, \ldots, A_{t}(t \geq 1)$ such that $A_{0}=A, A_{t}=B$, and $A_{r}$ is a contraction of $A_{r-1}$ for $r=1, \ldots, t[6]$.

Brualdi and Gibson [6] proved the following result about the permanent of a matrix. Lemma 1 LetA be a nonnegative integral matrix of order $n$ for $n>1$ and let $B$ be a contraction of A. Then

perA $=$ perB . 
Let $\mathrm{H}_{\mathrm{n}}=\mathrm{h}_{\mathrm{ij}}$ be an $\mathrm{n} \times \mathrm{n}$ pentadiagonal matrix as the following

$$
A=\left(\begin{array}{ccccccc}
1 & \mathrm{i} & -1 & 0 & \cdots & \cdots & 0 \\
-\mathrm{i} & 0 & \mathrm{i} & -1 & 0 & & \vdots \\
0 & -\mathrm{i} & 0 & \mathrm{i} & \ddots & \ddots & \vdots \\
\vdots & 0 & \ddots & \ddots & \ddots & \ddots & 0 \\
\vdots & & \ddots & \ddots & \ddots & \mathrm{i} & -1 \\
\vdots & & & 0 & -\mathrm{i} & 0 & \mathrm{i} \\
0 & \cdots & \cdots & \cdots & 0 & -\mathrm{i} & 0
\end{array}\right)_{\mathrm{n} \times \mathrm{n}}
$$

where $\mathrm{i}=\sqrt{-1}$. If $\mathrm{n}=5$, then we obtain the permanent of $\mathrm{H}_{5}$ by using Laplace

expansion as the following

$\operatorname{PerH}_{5}=\operatorname{Per}\left(\begin{array}{ccccc}1 & \mathrm{i} & -1 & 0 & 0 \\ -i & 0 & \mathrm{i} & -1 & 0 \\ 0 & -i & 0 & \mathrm{i} & -1 \\ 0 & 0 & -i & 0 & \mathrm{i} \\ 0 & 0 & 0 & -i & 0\end{array}\right)_{5 \times 5}$ 


$$
\begin{aligned}
& =\operatorname{Per}\left(\begin{array}{cccc}
0 & \mathrm{i} & -1 & 0 \\
-\mathrm{i} & 0 & \mathrm{i} & -1 \\
0 & -\mathrm{i} & 0 & \mathrm{i} \\
0 & 0 & -\mathrm{i} & 0
\end{array}\right)+(-1) \operatorname{Per}\left(\begin{array}{cccc}
\mathrm{i} & -1 & 0 & 0 \\
-\mathrm{i} & 0 & \mathrm{i} & -1 \\
0 & -\mathrm{i} & 0 & \mathrm{i} \\
0 & 0 & -\mathrm{i} & 0
\end{array}\right) \\
& =(-i) \operatorname{Per}\left(\begin{array}{ccc}
i & -1 & 0 \\
-i & 0 & i \\
0 & -i & 0
\end{array}\right)+\operatorname{Per}\left(\begin{array}{ccc}
0 & i & -1 \\
-i & 0 & i \\
0 & -i & 0
\end{array}\right)-\operatorname{Per}\left(\begin{array}{ccc}
-1 & 0 & 0 \\
-i & 0 & i \\
0 & -i & 0
\end{array}\right) \\
& =\operatorname{Per}\left(\begin{array}{cc}
0 & \mathrm{i} \\
-\mathrm{i} & 0
\end{array}\right)-\operatorname{Per}\left(\begin{array}{cc}
-1 & 0 \\
-\mathrm{i} & 0
\end{array}\right)+(-\mathrm{i}) \operatorname{Per}\left(\begin{array}{cc}
\mathrm{i} & -1 \\
-\mathrm{i} & 0
\end{array}\right)+\operatorname{Per}\left(\begin{array}{cc}
0 & \mathrm{i} \\
-\mathrm{i} & 0
\end{array}\right) \\
& =1-0+1+1=3=P(5) .
\end{aligned}
$$

By the contraction method introduced by Brualdi in [6], we now present the following theorem that gives the relationship between the permanent of the pentadiagonal matrix $\mathrm{H}_{\mathrm{n}}$ and the Padovan number $\mathrm{P}(\mathrm{n})$.

Theorem 2 Let $H_{n}$ be the $n \times n$ pentadiagonal matrix given by (2). Then the permanent of the matrix is equal to the $n^{\text {th }}$ Padovan number $P(n)$.

Proof.Let $\mathrm{H}_{\mathrm{n}}^{\mathrm{k}}$ be the $\mathrm{k}^{\text {th }}$ contraction of $\mathrm{H}_{\mathrm{n}}, 1 \leq \mathrm{k} \leq \mathrm{n}-2$. Since the definition of the matrix $\mathrm{H}_{\mathrm{n}}$; thematrix $\mathrm{H}_{\mathrm{n}}$ can be contracted on column 1 so that 


$$
\mathrm{H}_{\mathrm{n}}^{1}=\left(\begin{array}{ccccccc}
1 & 2 \mathrm{i} & -1 & 0 & \cdots & \cdots & 0 \\
-\mathrm{i} & 0 & \mathrm{i} & -1 & 0 & & \vdots \\
0 & -\mathrm{i} & 0 & \mathrm{i} & \ddots & \ddots & \vdots \\
\vdots & 0 & \ddots & \ddots & \ddots & \ddots & 0 \\
\vdots & & \ddots & \ddots & \ddots & \mathrm{i} & -1 \\
\vdots & & & 0 & -\mathrm{i} & 0 & \mathrm{i} \\
0 & \cdots & \cdots & \cdots & 0 & -\mathrm{i} & 0
\end{array}\right)_{(\mathrm{n}-1) \times(\mathrm{n}-1)}
$$

Since the matrix $\mathrm{H}_{\mathrm{n}}^{1}$ can be contracted on column 1

$$
\mathrm{H}_{\mathrm{n}}^{2}=\left(\begin{array}{ccccccc}
2 & 2 \mathrm{i} & -1 & 0 & \cdots & \cdots & 0 \\
-\mathrm{i} & 0 & \mathrm{i} & -1 & 0 & & \vdots \\
0 & -\mathrm{i} & 0 & \mathrm{i} & \ddots & \ddots & \vdots \\
\vdots & 0 & \ddots & \ddots & \ddots & \ddots & 0 \\
\vdots & & \ddots & \ddots & \ddots & \mathrm{i} & -1 \\
\vdots & & & 0 & -\mathrm{i} & 0 & \mathrm{i} \\
0 & \cdots & \cdots & \cdots & 0 & -\mathrm{i} & 0
\end{array}\right)_{(\mathrm{n}-2) \times(\mathrm{n}-2)}
$$

Furthermore, the matrix $\mathrm{H}_{\mathrm{n}}{ }^{2}$ can be contracted on column 1 and $\mathrm{P}(3)=\mathrm{P}(4)=2, \mathrm{P}(5)=3$ so that 


$$
\begin{aligned}
& \mathrm{H}_{\mathrm{n}}^{3}=\left(\begin{array}{ccccccc}
2 & 3 \mathrm{i} & -2 & 0 & \cdots & \cdots & 0 \\
-\mathrm{i} & 0 & \mathrm{i} & -1 & 0 & & \vdots \\
0 & -\mathrm{i} & 0 & \mathrm{i} & \ddots & \ddots & \vdots \\
\vdots & 0 & \ddots & \ddots & \ddots & \ddots & 0 \\
\vdots & & \ddots & \ddots & \ddots & \mathrm{i} & -1 \\
\vdots & & & 0 & -\mathrm{i} & 0 & \mathrm{i} \\
0 & \cdots & \cdots & \cdots & 0 & -\mathrm{i} & 0
\end{array}\right)_{(\mathrm{n}-3) \times(\mathrm{n}-3)} \\
& =\left(\begin{array}{ccccccc}
\mathrm{P}(4) & \mathrm{iP}(5) & -\mathrm{P}(3) & 0 & \cdots & \cdots & 0 \\
-\mathrm{i} & 0 & \mathrm{i} & -1 & 0 & & \vdots \\
0 & -\mathrm{i} & 0 & \mathrm{i} & \ddots & \ddots & \vdots \\
\vdots & 0 & \ddots & \ddots & \ddots & \ddots & 0 \\
\vdots & & \ddots & \ddots & \ddots & \mathrm{i} & -1 \\
\vdots & & & 0 & -\mathrm{i} & 0 & \mathrm{i} \\
0 & \cdots & \cdots & \cdots & 0 & -\mathrm{i} & 0
\end{array}\right)_{(\mathrm{n}-3) \times(\mathrm{n}-3)}
\end{aligned}
$$

Continuing this process, we have 


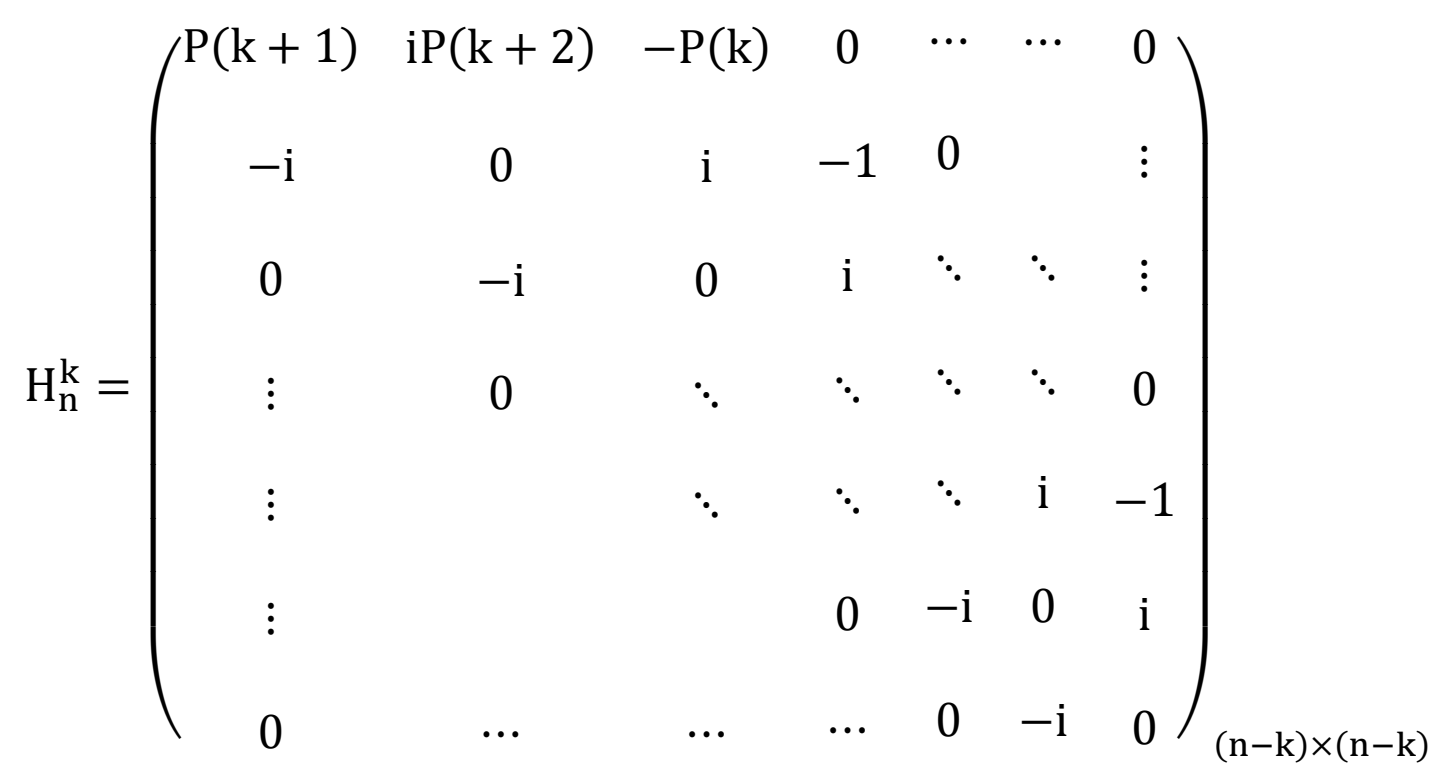

for $3 \leq \mathrm{k} \leq \mathrm{n}-4$. Hence,

$$
\mathrm{H}_{\mathrm{n}}^{\mathrm{n}-3}=\left(\begin{array}{ccc}
\mathrm{P}(\mathrm{n}-2) & \mathrm{iP}(\mathrm{n}-2) & -\mathrm{P}(\mathrm{n}-3) \\
-\mathrm{i} & 0 & \mathrm{i} \\
0 & -\mathrm{i} & 0
\end{array}\right)_{3 \times 3}
$$

which, by contraction of $\mathrm{H}_{\mathrm{n}}^{\mathrm{n}-3}$ on column 1 , gives

$$
\mathrm{H}_{\mathrm{n}}^{\mathrm{n}-2}=\left(\begin{array}{cc}
\mathrm{P}(\mathrm{n}-1) & \mathrm{iP}(\mathrm{n}) \\
-\mathrm{i} & 0
\end{array}\right)_{2 \times 2}
$$

By applying equation (1), we obtain $\operatorname{perH}_{n}=\mathrm{H}_{\mathrm{n}}^{\mathrm{n}-2}=-\mathrm{i}^{2} \mathrm{P}(\mathrm{n})=\mathrm{P}(\mathrm{n})$ which is desired

2.1. Maple Procedure

The following Maple procedure calculates the permanent of the pentadiagonal matrix $\mathrm{H}_{\mathrm{n}}$ given by (2).

restart:

with(LinearAlgebra):

permanent:=proc $(\mathrm{n})$ 
local I,j,r,f,H;

$\mathrm{f}:=(\mathrm{i}, \mathrm{j})->$ piecewise $(\mathrm{i}=1$ and $\mathrm{j}=1,1, \mathrm{j}-\mathrm{i}=-1,-\mathrm{I}, \mathrm{j}-\mathrm{i}=1, \mathrm{I}, \mathrm{j}-\mathrm{i}=2,-1,0)$;

$\mathrm{H}:=\operatorname{Matrix}(\mathrm{n}, \mathrm{n}, \mathrm{f})$ :

for $r$ from 0 to $n-2$ do

$\operatorname{print}(\mathrm{r}, \mathrm{H})$ :

for $\mathrm{j}$ from 2 to $\mathrm{n}-\mathrm{r}$ do

$\mathrm{H}[1, \mathrm{j}]:=\mathrm{H}[2,1]^{*} \mathrm{H}[1, \mathrm{j}]+\mathrm{H}[1,1] * \mathrm{H}[2, \mathrm{j}]$ :

od:

H:=DeleteRow(DeleteColumn(Matrix(n-r,n-r,H), 1),2):

od:

$\operatorname{print}(\mathrm{r}, \mathrm{eval}(\mathrm{H}))$ :

end proc:with(LinearAlgebra):

permanent(n);

\section{References}

[1] T. Koshy, Fibonacci and Lucas Numbers with Applications, Wiley-Interscience, New York, 2001.

[2] T. Koshy, Fibonacci, Lucas, and Pell numbers, and Pascal's triangle, Mathematical Spectrum 43(3) (2011), 125-132.

[3] A. G. Shannon, P. G. Anderson, A. F. Horadam, Properties of Cordonnier, Perrin and Van der Laan numbers, International Journal of Mathematical Education in Science and Technology 37 (2006), 825-831.

[4] The OEIS Foundation Inc., The On-Line Encyclopedia of Integer Sequences, http://oeis.org, 2013.

[5] H. Minc, Permanents, Encyclopedia of mathematics and its applications, Addison-Wesley, New York, 1978.

[6] R. A. Brualdi, P. M. Gibson, Convex polyhedra of doubly stochastic matrices I: applications of the permanent function, J. Combin. Theory A 22 (1977), 194-230.

[7] R. A. Brualdi, D. Cvetkovic, A Combinatorial Approach to Matrix Theory and Its Applications, CRC Press, 2009.

[8] F. Harary, Determinants, permanents and bipartite graphs, Mathematics Magazine 42 (1969) 146-148.

[9] M. Marcus, H. Minc, Permanents, American Mathematical Monthly 72 (1965) 577-591. 
[10] G. Y. Lee, S. G. Lee, and H. G. Shin, On the k-generalized Fibonacci matrix $Q_{k}$, Lin. Alg. Appl. 251 (1997) 73-88.

[11] G. Y. Lee, k-Lucas numbers and associated bipartite graphs, Lin. Alg. Appl. 320 (2000), 51-61.

[12] W. C. Shiu and Peter C. B. Lam, More on the generalized Fibonacci numbers and associated bipartite graphs, Int. Math. J. 3 (2003), 5-9.

[13] E. K1lic, and D. Tasc1, On families of bipartite graphs associated with sums of Fibonacci and Lucas numbers, Ars Combin. 89 (2008), 31-40.

[14] G. Y. Lee and S. G. Lee, A note on generalized Fibonacci numbers, Fibonacci Quart. 33 (1995), 273-278.

[15] E. K1lic, and D. Tasc1, On the permanents of some tridiagonal matrices with applications to the Fibonacci and Lucas numbers, Rocky Mt. J. Math. 37 (2007), 19531969.

[16] M. Akbulak and A. Oteles, On the number of 1-factors of bipartite graphs, Math. Sci. Lett. 2 (2013), 1-7.

[17] A. Oteles, On the number of perfect matchings for some certain types of bipartite graphs, Filomat 31(2017), 4809-4818.

[18] K. Kaygisiz and A. Sahin, Determinants and permanents of Hessenberg matrices and generalized Lucas polynomials, Bull. Iranian Math. Soc. 39 (2013), 1065-1078.

[19] F. Y1lmaz and D. Bozkurt, The adjacency matrix of one type of directed graph and the Jacobsthal numbers and their determinantal representation, J. Appl. Math. 2012 (2012), 1-14

[20] F. Y1lmaz and D. Bozkurt, Some properties of Padovan sequence by matrix methods, Ars Combin. 104(2012), 149-160.

[21] C. M. da Fonseca, T. Sogabe, and F. Y1lmaz, Lower k-Hessenberg matrices and k-Fibonacci, Fibonacci-p and Pell (p, i) number, Gen. Math. Notes 31 (2015), 10-17.

[22] E. K1lic, and D. Tasc1, On families of bipartite graphs associated with sums of generalized order-k Fibonacci and Lucas numbers, Ars Combin. 94 (2008), 13-23.

[23] E. K1lıc, and A. P. Stakhov, On the Fibonacci and Lucas p-numbers, their sums, families of bipartite graphs and permanents of certain matrices, Chaos Solitons Fractals 40 (2009), 10-21.

[24] P. Vasco, P. Catarino, H. Campos, A. P. Aires, and A. Borges, k-Pell, k-PellLucas and modified k-Pell numbers: Some identities and norms of Hankel matrices, Int. J. Math. Anal. 9 (2015), 31-37. 